\title{
Computing C1s X-ray absorption for Single-Walled Carbon Nanotubes with distinct electronic type
}

\author{
D.J. Mowbray, ${ }^{1}$ P. Ayala, ${ }^{2}$ T. Pichler, ${ }^{2}$ and A. Rubio ${ }^{1,3}$ \\ ${ }^{1}$ Nano-bio Spectroscopy Group and ETSF Scientific Development Center, \\ Departamento de Física de Materiales, Universidad del País Vasco, \\ Centro de Física de Materiales CSIC-UPV/EHU-MPC and DIPC, Avenida de Tolosa 72, E-20018 San Sebastián, Spain \\ ${ }^{2}$ University of Vienna, Faculty of Physics, 1090 Wien, Austria \\ ${ }^{3}$ Fritz-Haber-Institut der Max-Planck-Gesellschaft, Berlin, Germany
}

\begin{abstract}
The experimental data recorded for the $\mathrm{C} 1 s$ edge in X-ray absorption spectroscopy (XAS) for graphite, graphene and nanotubes, have consistently exhibited certain discrepancies when compared with theoretical calculations. A theoretical approach to estimate the energy scale normalization for the $\mathrm{C} 1 s$ shape in X-ray absorption is presented within the time dependent density functional theory and random phase approximation framework employing the loss function. The position of the $\sigma$ resonance is fairly localized whereas core hole effects must be envisaged in order to have an agreement with the delocalization of the $\pi^{*}$ resonance, which is displaced by $\sim 2 \mathrm{eV}$. Here we report a combined experimental and theoretical approach to identify the electronic conduction band of single walled carbon nanotubes using XAS, taking into account their metallic character.
\end{abstract}

Keywords: nanotubes, X-ray absorption, NEXAFS, TDDFT, metallicity sorting

\section{INTRODUCTION}

Carbon based molecular structures have been the focus of a great amount of research in the last decades, as demonstrated by their many potential applications, and the awarding of two Nobel prizes in the area (for fullerenes and graphene). Furthermore, the area of single-walled carbon nanotubes (SWCNTs), represents a rich playground to study several fundamental physics problems [1]. The structural, mechanical and electronic properties of SWCNTs are fully determined by the geometrical C atom arrangement. Given this peculiarity, methods within solid state spectroscopy have been developed towards the evaluation of these properties. In this context, for many years carbon based structures have been widely studied by $\mathrm{X}$-ray absorption spectroscopy (XAS), which is a very useful bulk probing method that allows one to both examine the unoccupied density of states and identify different site selective bonding environments (see figure 1) [2-7].

Experimentally, the two most well known spectral features in the XAS core level spectrum are the $\sigma^{*}$ and $\pi^{*}$ bands. Both have been analyzed in several studies for graphitic systems (see the overall spectrum in the inset of figure 1). However, in the case of SWCNTs, which are structures with unique onedimensional electronic properties, additional features in the core level XAS spectrum have only recently been revealed experimentally [3, 8,-10]. In this article we outline the $\mathrm{C} 1 s$ response in XAS, which is again related to the density of states of the conduction band in SWCNTs and its fine structure revealed by experiments performed specifically on metallicitysorted SWCNT material [11].

At this point it is worth mentioning that the experimental data recorded for the $\mathrm{C} 1 s$ edge in X-ray absorption spectroscopy for graphite, graphene and nanotubes, has consistently exhibited certain theoretical limitations the moment theoretical calculations and experiments are compared. For instance, the electronic structure of graphite has been widely studied experimentally and theoretically and the $\sigma^{*}$ and $\pi^{*}$ thresholds have been examined by various authors [4, 5, 12, [13]. Theoretically, an intense debate existed around a discrep- ancy of the position of a peak appearing $3 \mathrm{eV}$ above the Fermi level $\left(\varepsilon_{\mathrm{F}}\right)$ in the ground state density of states (DOS), whereas experimental XAS observations found that peak shifted to only $1 \mathrm{eV}$ above the $\mathrm{C} 1 s$ onset. This peak is associated to the $\pi^{*}$ resonance and its dislocation has been found to be strongly affected by excitonic effects from the hole in the core $\mathrm{C} 1 s$ level.

In order to resolve XAS spectra with a clear fine structure, we have taken advantage of the positive developments that have been made controlling and optimizing the synthesis, purification and separation of SWCNTs by metallicity and chirality. We have performed our experiments with films with a characteristic narrow Gaussian diameter distribution centered at $13.7 \AA$ with a spread of $0.8 \AA$ [14]. The quality of these samples is backed up by photoemission studies described in reference [8], where the disentanglement of the van Hove singularities (vHs) of metallic and semiconducting nanotubes were reported. There, the vHs associated to specific types of tubes were approximated by a simple diameter cumulative tight binding calculation to estimate their relative positions for tubes corresponding to the same diameter range. Most of the earliest calculations addressing the electronic structure of graphite were done taking into account the local density approximation (LDA). Tight binding (TB) calculations were later performed and improved considering the DOS in the presence of a core hole. The theoretical work related to the $\mathrm{C} 1 s$ absorption edge in carbon nanotubes is much more limited. Our interest here is to use a theoretical approach to estimate the energy scale normalization for the $\mathrm{C} 1 s$ shape in XAS for nanotubes in the diameter range, for which a defined and characteristic fine structure in the $\pi^{*}$ resonance has been encountered.

In order to do this, we have used an alternative method to previous core-hole calculations, which involves the use of density functional theory (DFT) to calculate the loss function $-\Im\left\{\varepsilon^{-1}(\mathbf{q}, \omega)\right\}$ within the time dependent DFT and random phase approximation (TDDFT-RPA) framework. The position of the $\sigma^{*}$ resonance results are fairly localized whereas core hole effects must be envisaged in order to have an agreement 


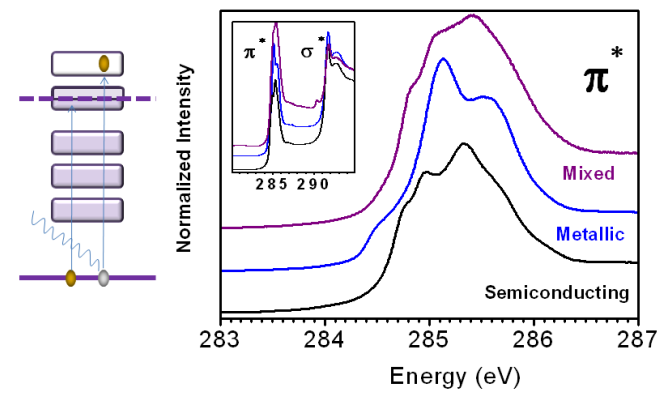

FIG. 1. C1s near edge X-ray absorption in the $\sigma^{*}$ and $\pi^{*}$ regions for SWCNTs with random metallicity and sorted species. The right panel shows a close-up to the $\pi^{*}$ resonance revealing its fine structure, which is a fingerprint of the corresponding samples.

with the delocalization of the $\pi^{*}$ resonance. This is displaced around $2 \mathrm{eV}$ in agreement with early work reported by Mele and Ritsko [15] and later by Ahuja et al. [5]. Additionally, we show how spectral broadening of the individual electronic states is associated to the fine structure in the $\pi^{*}$ line and how they influence its line shape.

\section{EXPERIMENTAL METHODOLOGY}

The nanotubes used for the measurements presented herein were synthesized by arc discharge, purified and later separated by density gradient ultracentrifugation [14]. Filtration was then used to prepare films of SWCNTs on sapphire plates and they were later annealed above $800 \mathrm{~K}$ in ultra high vacuum. The high purity samples have a Gaussian diameter distribution centered at $13.7 \AA$ with a spread of $0.8 \AA$ [14, 16]. The XAS experiments were carried out at the beamline UE52PGM at BESSY II and all spectra were recorded in partial yield mode and bulk sensitive drain current.

\section{THEORETICAL METHODOLOGY}

In order to obtain the best possible agreement between the calculations to the experiments, we use nanotubes that are in the experimental samples' diameter distribution range. Keeping in mind that we have analyzed material after metallicity sorting, ideal candidates for this study are the SWCNTs with $(10,10)$ and $(17,0)$ chiralities.

All DFT calculations were performed using the real-space projector-augmented-wave (PAW) method code GPAW [17, [18], with a grid spacing of $0.2 \AA$, and the Perdew-BurkeErnzerhof exchange correlation (xc)-functional [19]. An electronic temperature of $k_{B} T \approx 0.05 \mathrm{eV}$ was used to obtain the occupation of the Kohn-Sham orbitals, with all energies extrapolated to $T=0 \mathrm{~K}$, and one unoccupied band per $\mathrm{C}$ atom included to improve convergence. Periodic boundary conditions were employed along the SWCNT axes with Monkhorst-
Pack $k$-point samplings of 5 and 9 for the $(17,0)$ zigzag and $(10,10)$ armchair SWCNTs, respectively.

Structural minimization was performed within the Atomic Simulation Environment (ASE), until a maximum force below $0.05 \mathrm{eV} / \AA ̊$ was obtained. The resulting SWCNT diameters compared with the values obtained geometrical formula $d_{\varnothing} \approx \frac{a}{\pi} \sqrt{n^{2}+n m+m^{2}}$ are shown in Table II The opti-

TABLE I. $(10,10)$ and $(17,0)$ SWCNT diameters from DFT calculated with the PBE xc-functional $d_{\mathrm{PBE}}$ are compared with the geometrical formula $d_{\varnothing} \approx \frac{a}{\pi} \sqrt{n^{2}+n m+m^{2}}$, where $a \approx 2.46 \AA$.

\begin{tabular}{|c|c|c|c|c|}
\hline SWCNT & Type & $d_{\mathrm{PBE}}$ & $d_{\varnothing}$ & Atoms/Cell \\
\hline$(10,10)$ armchair & Metallic & $13.63 \AA$ & $13.56 \AA$ & 40 \\
$(17,0)$ zigzag & Semiconducting & $13.39 \AA$ & $13.31 \AA$ & 68 \\
\hline
\end{tabular}

mized zigzag and armchair SWCNT minimal unit cells were $4.272 \AA$ and $2.470 \AA$, respectively, in the axial $z$-direction and 24 Åin the perpendicular $x$ and $y$ directions, as shown schematically in figure 2. Perpendicular to the SWCNT-axis the unit cells were made non-periodic, with the wave functions and densities set to zero at the boundaries to model isolated SWCNTs.

To ensure a sufficient resolution of the wave functions in momentum space for the core-hole and TDDFT-RPA calculations, the electronic structure was re-optimized with 25 and $15 k$-points in the axial direction for the $(10,10)$ and $(17,0)$ SWCNTs, respectively. This yielded a resolution in $k$-space of $\Delta k \approx 0.1 \AA^{-1}$. We have also employed a broadening of $\eta=0.5 \mathrm{eV}$ in both the core-hole and TDDFT-RPA calculations, as has previously used when describing the loss function of graphite [20]. To reduce interactions between neighboring core-holes, calculations for the armchair SWCNTs were performed with a repeated unit cell, yielding a separation of $4.940 \AA$ between repeated images. The X-ray absorption cross section $\left\langle\psi_{n}|\mathbf{r}| \phi^{a}\right\rangle$ was then calculated using a half core-hole on the C1s level within the PAW method. Here $\psi_{n}$ are the Kohn-Sham all-electron wave functions calculated within the PAW methodology, $\phi^{a}$ is the $\mathrm{C} 1 s$ atomic orbital, which is half occupied, and $\mathbf{r}$ is in the direction of excitation.

To obtain the C1s XAS response within the TDDFT-RPA methodology, we have first performed all-electron calculations, including explicitly the core $\mathrm{C} 1 s$ states at the DFT level within the PAW methodology and the frozen-core approximation. The loss function $-\Im\left\{\varepsilon^{-1}(\mathbf{q}, \omega)\right\}$ was then calculated using linear response TDDFT-RPA, as implemented in the real-space PAW code GPAW [21], with local field effects included.

In this way, one may include the core states as Kohn-Sham wave functions at the self-consistent step of the DFT calculation. This allows us to obtain the XAS response directly within the random phase approximation from $\mathbf{q} \cdot\left\langle\psi_{1 s}|\vec{\nabla}| \psi_{n}\right\rangle$. In other words, all the electrons have been included within the frozen-core approximation, meaning that the core $1 s$ orbitals are included when calculating the TDDFT-RPA loss function, yielding the XAS C1s spectrum. 


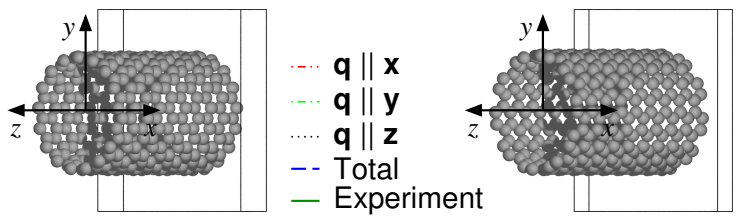

(a) $(17,0)$ SWCNT

(b) $(10,10)$ SWCNT

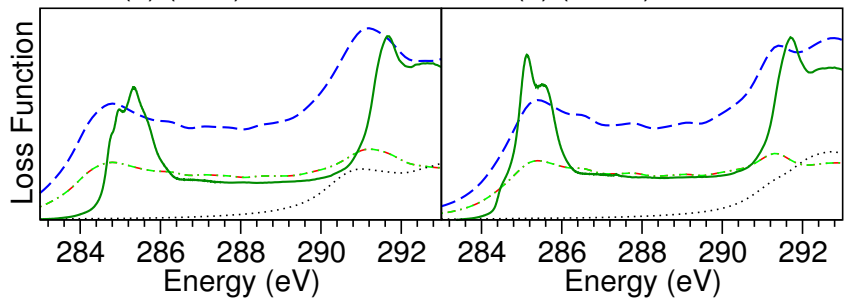

FIG. 2. Loss function for (a) a $(17,0)$ semiconducting zigzag SWCNT and (b) a $(10,10)$ metallic armchair SWCNT, versus energy in $\mathrm{eV}$ from XAS measurements (- - , and TDDFT-RPA calculations in the $x(-\cdot \cdot-\cdot)$ ) and $y(-\cdot-\cdot-\cdot)$ directions perpendicular to the SWCNT axis, the $z$ direction $(\cdots \cdots)$ parallel to the SWCNT axis, and the total response (- $\left.-\_\right)$, as shown schematically in the upper panels.

\section{RESULTS AND DISCUSSION}

As described in the introductory section, the nature of the $\pi^{*}$ resonance in carbon systems has always exhibited discrepancies between theory and experiment. We address this problem for SWCNTs, by taking into account the fine structure observed in the $\pi^{*}$ spectra recorded experimentally for tubes with a narrow diameter distribution. The XAS C1s spectrum for SWCNTs in this diameter range is shown in figure 1 The inset shows the overall $\mathrm{C} 1 s$ response, whereas the main panel shows a close-up to the $\pi^{*}$ response, where the fine structure for purely semiconducting and purely metallic SWCNT-films can be observed. Even in the case of random metallicity samples, a fine structure is revealed in the top spectrum. This fine structure is due to the molecular like response of the vHs of the corresponding SWCNTs which are polarized perpendicular to the tube axis, as will be explained later.

We have performed two types of calculations in order to identify the nature of this fine structure. In Ref. [8] we proved, by using the metallicity sorted SWCNT with a mean diameter of $13.7 \AA$, that this fine structure is related to the vHs of the individual SWCNTs. In that contribution we used diameter cumulative TB calculations of the electronic structure, and found a perfect match to the experimental spectra. However, those calculations include scaling parameters and are semi-empirical. Therefore, we decided to perform $a b$ initio calculations to validate our semi-empirical fit. To be consistent with the previous results and to keep a reasonable timescale, archetypal examples of SWCNTs with a mean diameter as close as possible to $13.7 \AA$ were chosen $(\mathrm{a}(17,0)$ semiconducting zigzag SWCNT and a $(10,10)$ metallic armchair SWCNT).

In figure 2 we show a schematic of how the calculations using the loss function have been performed, considering momentum transfer perpendicular $(z)$ and along the tube axis $(x, y)$. The perpendicular component has a larger contribution to the $\pi^{*}$ peak, while momentum transfers along the SWCNT axis contribute to the higher energy $\sigma^{*}$. In the lower panel an overall comparison of the all electron loss function calculations with the experimental data is provided. In each case the onset for the XAS is shifted to the difference in total energy between the ground state of the carbon nanotube, and the total energy of the SWCNT with a full hole in the $1 s$ level and an excited electron in the conduction band. This gives a value for the onset at the $\Gamma$-point. To correct the onset for the loss function calculations, a scissors operator on the energy difference at the $\Gamma$-point between the lowest unoccupied molecular orbital and the top of the $1 s$ band in the ground state calculation for the SWCNT, has been used. (a) $(10,10)$ SWCNT

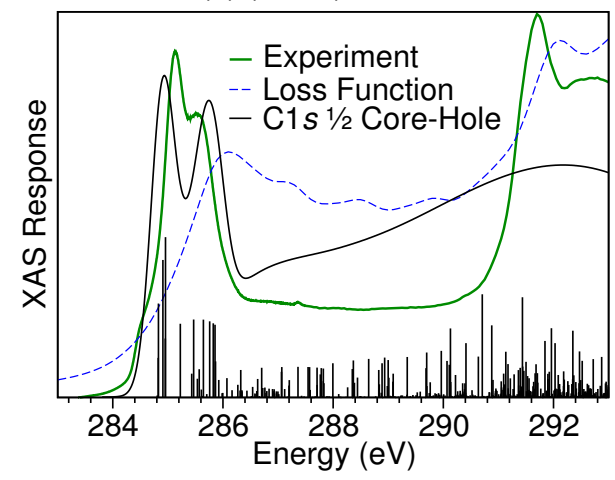

(b) $(17,0)$ SWCNT

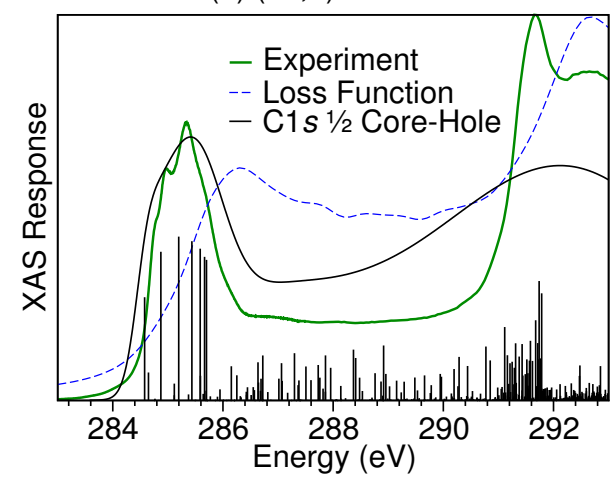

FIG. 3. XAS C1 $s \pi^{*}$ absorption edge of SWCNTs with (a) metallic and (b) semiconducting species (—) are compared with loss function $-\Im\left\{\varepsilon^{-1}(\mathbf{q}, \omega)\right\}$ calculations within TDDFT-RPA (- - -) and $1 / 2$ core-hole calculations $\left\langle\psi_{n}|\mathbf{r}| \phi^{a}\right\rangle$ (—) for (a) $(10,10)$ and (b) $(17,0)$ SWCNTs. The calculated spectra have been shifted to align the $\mathrm{C} 1 s$ onset with experiment, by (a) $\Delta \varepsilon \approx+0.7 \mathrm{eV}$ for the $(10,10)$ SWCNT, and (b) $\Delta \varepsilon \approx+1.2 \mathrm{eV}$ for the $(17,0)$ SWCNT. The black vertical lines denote the eigenenergies and intensities of the transitions between the $n^{\text {th }}$ Kohn-Sham wave function $\psi_{n}$ and the C $1 s$ half-occupied atomic orbital $\phi^{a}$.

In figure 3 core-hole calculations for $(10,10)$ and $(17,0)$ SWCNTs are compared with the all electron TDDFT-RPA loss function calculations. The spectra obtained experimentally are also depicted in both cases. We observe that the onset for the XAS spectra is responsible for the major spectral discrepancies. To calculate the onset for the first excited orbital, 
two DFT ground state calculations at the $\Gamma$-point have been done: One using the regular frozen core for carbon, and the other using a full core-hole in one of the carbon atoms, adding the electron to the valence, and setting the magnetic moment of the system to +1 . The total energy difference between these two systems was taken, which gives the energy cost for taking one electron from the core of a carbon atom the SWCNT and putting it in the lowest unoccupied molecular orbital at the $\Gamma$ point. The black vertical lines denote the eigenenergies of the calculated Kohn-Sham orbitals. This yielded C1s onset energies within $0.5 \%$ of the experimental values. In Fig. 3 we have removed the remaining discrepancy by shifting the theoretical $\mathrm{C} 1 s$ onset energies up by $0.7 \mathrm{eV}$ and $1.5 \mathrm{eV}$ for the $(10,10)$ and $(17,0)$ SWNTs, respectively.

For the core-hole calculation, if we align the position of the first peak the results are quite accurate. The problem encountered in the mismatching of the loss function calculation seems also to be affected by the number of atoms involved in the calculation. However, we will not discuss this matter in this contribution as it involves other calculations with smaller diameter tubes, which we do not aim at describing here. In any case, we find a good agreement regarding the fine structures, which have a long lifetime yielding sharp and distinct peaks on the XAS response perpendicular to the tube axis and on the other hand to the broad $\pi^{*}$ resonance along the tube axis having a strong lifetime broadening like in graphite. This is in good agreement with the two fold XAS response we observed in our previous experimental report [8] where we attributed this fine structure to the molecular like response of the vHs of the corresponding SWCNTs which are polarized perpendicular to the tube axis. The overall shape of the $\mathrm{C} 1 s \pi^{*}$ peak shows close similarities to the $\pi^{*}$ resonance of bulk $s p^{2}$ carbon in graphite or graphene. We therefore assigned the spectra of nanotubes to a clear twofold response in the XAS from excitations parallel (graphene like) and perpendicular (molecule like) to the tube axis, which have different core hole effects.

In figure 4 the spectrum corresponding to a metallic $(10,10)$ SWCNT is shown. In both figures the gray line represents the experimental data, whereas the blue line corresponds to the result of each fit. The fit shown in figure 4(a) has been done using the theoretical result from the core-hole calculations shown in figure 3 using a broadening of $\eta=0.08 \mathrm{eV}$ to consider the lifetime effects. The fit in figure 4 (b) corresponds to a diameter cumulative TB DOS broadened by the experimental resolution. The semi-empiric diameter cumulative TB calculations presented in Ref. [8] agree well with the results we show here. Of course, these new calculations have a better match regarding the relative intensity of the individual vHs related peaks. These results also confirm that the core hole lifetime is strongly orientation dependent and much larger perpendicular than parallel to the tube axis. Additionally, it exhibits a similar lifetime to the graphite case.

\section{CONCLUSIONS}

A combined experimental and theoretical approach to identify the electronic conduction band of single walled carbon
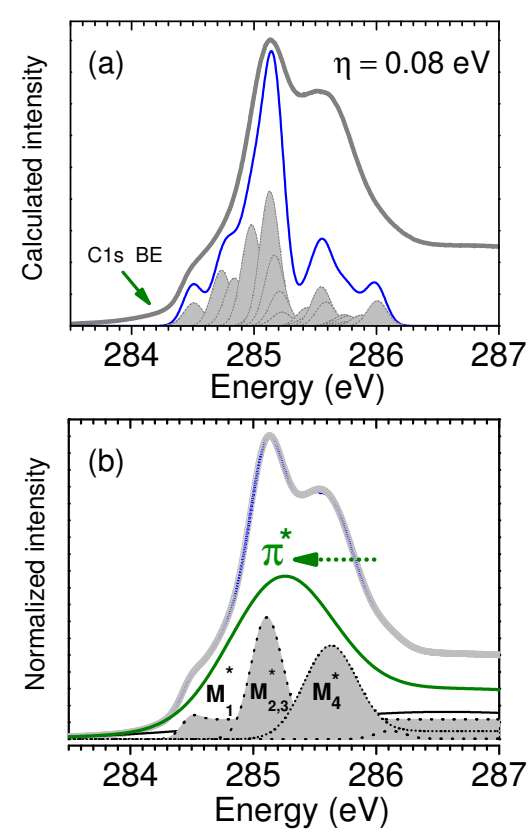

FIG. 4. (a) Spectrum of metallic SWCNTs obtained experimentally compared to the calculated transitions obtained with relative intensity given by $\left\langle\psi_{n}|\mathbf{r}| \phi^{a}\right\rangle$ for a $(10,10)$ SWCNT. A broadening of $\eta=$ $0.08 \mathrm{eV}$ has been used to consider the life time effects. (b) Close up of the high resolution XAS C1s $\pi^{*}$ absorption edge (circles) of metallic SWCNTs (same experimental data as in (a)), together with a line shape analysis. This depicts the diameter cumulative TB DOS broadened by the experimental resolution. The labels denote the vHs $\left(\mathrm{M}_{1 . .4}^{*}\right)$ and the overall conduction band $\pi^{*}$.

nanotubes using XAS taking into account their metallic character has been reported. Two types of calculations have been performed to obtain the $\mathrm{C} 1 s$ XAS response for SWCNTs. A theoretical approach to estimate the energy scale normalization for the $\mathrm{C} 1 s$ shape in X-ray absorption has been used, employing DFT calculations of the loss function within the TDDFT-RPA framework. The position of the $\sigma^{*}$ resonance is fairly localized whereas core hole effects must be envisaged in order to have an agreement with the delocalization of the $\pi^{*}$ resonance, which is displaced by $\sim 2 \mathrm{eV}$. This is in good agreement with previous studies in graphite and contributes to the experimental observations that suggest a twofold response in the case of SWCNTs where: The core hole effect in the broad $\mathrm{C} 1 s \pi^{*}$ resonance just resembles bulk $s p^{2}$ carbon, and the diminutive core hole effects in the resonances due to $\mathrm{vHs}$ are typical for molecular excitations.

\section{ACKNOWLEDGEMENT}

This work was supported by the Austrian Science Fund through project FWF P21333-N20. We acknowledge the technical assistance from S. Leger and R. Hübel from the IFWDresden. We acknowledge the Helmholtz-Zentrum Berlin- 
Electron storage ring BESSY II for provision of synchrotron radiation at beamline UE52PGM. The research leading to these results has received funding from the EU (FP7/20072013) under grant agreement No. 226716. P.A. was supported by a Marie Curie Intra European Fellowship within the 7th European Community Framework Programme. A.R. acknowledges the support of the European Research Coun- cil Advanced Grant DYNamo (ERC-2010-AdG -Proposal No. 267374), Spanish MICINN (FIS2010-21282-C02-01), "Grupos Consolidados UPV/EHU del Gobierno Vasco" (IT-31907), ACI-Promociona (ACI2009-1036) and European Community e-I3 ETSF project (Contract No. 211956). D.J.M. acknowledges funding through the Spanish "Juan de la Cierva" program (JCI-2010- 08156).
[1] A. Jorio, M. Dresselhaus, and G. Dresselhaus, Carbon Nanotubes: Advanced Topics in the Synthesis, Structure, Properties and Applications (Springer-Verlag, Heidelberg, 2008).

[2] P. E. Batson, Phys. Rev. B, 48, 2608 (1993).

[3] C. Kramberger, H. Rauf, H. Shiozawa, M. Knupfer, B. Buchner, T. Pichler, D. Batchelor, and H. Kataura, Phys. Rev. B., 75, 235437 (2007).

[4] X. Weng, P. Rez, and H. Ma, Phys. Rev. B, 40, 4175 (1989).

[5] R. Ahuja, P. A. Bruhwiler, J. M. Wills, B. Johansson, N. Martensson, and O. Eriksson, Phys. Rev. B, 54, 14396 (1996).

[6] O. Plashkevych, T. Privalov, H. Agren, V. Carravetta, and K. Ruud, Chem. Phys., 260, 11 (2000).

[7] S. G. Urquhart, H. Ade, M. Rafailovich, J. S. Sokolov, and Y. Zhang, Chem. Phys. Lett., 322, 412 (2000).

[8] P. Ayala, Y. Miyata, K. De Blauwe, H. Shiozawa, Y. Feng, K. Yanagi, C. Kramberger, S. R. P. Silva, R. Follath, H. Kataura, and T. Pichler, Physical Review B, 80 (2009), ISSN 1098-0121.

[9] P. Ayala, H. Shiozawa, K. De Blauwe, Y. Miyata, R. Follath, H. Kataura, and T. Pichler, Journal of Materials Science, 45, 5318 (2010).

[10] K. De Blauwe, D. J. Mowbray, Y. Miyata, P. Ayala, H. Shiozawa, A. Rubio, P. Hoffmann, H. Kataura, and T. Pichler, Phys. Rev. B., 82 (2010).

[11] M. S. Arnold, A. A. Green, J. F. Hulvat, S. I. Stupp, and M. C. Hersam, Nat. Nanotec., 1, 60 (2006).
[12] P. A. Bruhwiler, P. Kuiper, O. Eriksson, R. Ahuja, and S. Svensson, Phys. Rev. Lett., 76, 1761 (1996).

[13] R. V. Vedrinskii, V. L. Kraizman, A. A. Novakovich, G. Y. Machavariani, and V. S. Machavariani, Journal of Physics: Condensed Matter, 6, 11045 (1994).

[14] Y. Miyata, K. Yanagi, Y. Maniwa, and H. Kataura, J. Phys. Chem. C, 112, 13187 (2008).

[15] E. J. Mele and J. J. Ritsko, Phys. Rev. Lett., 43, 68 (1979).

[16] Z. Wu, Z. Chen, X. Du, J. M. Logan, J. Sippel, M. Nikolou, K. Kamaras, J. R. Reynolds, D. B. Tanner, A. F. Hebard, and A. G. Rinzler, Science, 305, 1273 (2004).

[17] J. Mortensen, L. Hansen, and K. Jacobsen, Phys. Rev. B, 71, 035109 (2005).

[18] J. Enkovaara, C. Rostgaard, J. Mortensen, J. Chen, M. Dulak, L. Ferrighi, J. Gavnholt, C. Glinsvad, V. Haikola, H. Hansen, H. Kristoffersen, M. Kuisma, A. Larsen, L. Lehtovaara, M. Ljungberg, O. Lopez-Acevedo, P. Moses, J. Ojanen, T. Olsen, V. Petzold, N. Romero, J. Stausholm-Møller, M. Strange, G. Tritsaris, M. Vanin, M. Walter, B. Hammer, H. Häkkinen, G. Madsen, R. Nieminen, J. Nørskov, M. Puska, T. Rantala, J. Schiøtz, K. S. Thygesen, and K. W. Jacobsen, Journal of Physics: Condensed Matter, 22, 253202 (2010).

[19] J. P. Perdew, K. Burke, and M. Ernzerhof, Phys. Rev. Lett., 77, 3865 (1996).

[20] A. G. Marinopoulos, L. Reining, V. Olevano, A. Rubio, T. Pichler, X. Liu, M. Knupfer, and J. Fink, Phys. Rev. Lett. 89, 076402 (2002).

[21] J. Yan, J. Mortensen, K. Jacobsen, and K. Thygesen, Phys.Rev. B, 83 (2011). 